\title{
Espondilodiscitis tuberculosa en Cádiz (España) durante diez años
}

\author{
MARÍA CARMEN LOZANO ${ }^{1}$, LIDIA GARCÍA-AGUDO ${ }^{1}$, RAQUEL MORENO ${ }^{1}$, \\ NICOLÁS CHOZAS ${ }^{2}$, PEDRO GARCÍA-MARTOS ${ }^{1}$
}

'Microbiología.

${ }^{2}$ Reumatología. Hospital Universitario Puerta del Mar. Cádiz, España.

Recibido el 18 de marzo de 2010, aceptado el 26 de julio de 2010.

Correspondencia a: Pedro García-Martos Avda. Ana de Viya, $13-2^{\circ} \mathrm{B}$ 11009 Cádiz (España). Tfno: +34956003068 Fax: +34956003081 E-mail: pedromartos@hotmail. a tuberculosis osteoarticular representa hasta un tercio de los casos de tuberculosis extrapulmonar ${ }^{1}$. En los países industrializados afecta predominantemente a adultos, mientras que en los países en vías de desarrollo incide sobre todo en niños y jóvenes ${ }^{2,3}$. Suele producirse por vía hematógena, aunque también es posible la vía linfática o por contigüidad desde otro foco ${ }^{1,4}$. Aproximadamente, $30 \%$ de los pacientes presenta evidencia radiológica de enfermedad pulmonar y un porcentaje algo inferior, infección del tracto genitourinario, permaneciendo sin identificar el foco primario en más de la mitad de los casos. Con frecuencia, existe un período de latencia prolon- gado (media de 12-18 meses) entre el episodio de infección pulmonar y el desarrollo de manifestaciones músculo-esqueléticas ${ }^{4}$.

La espondilodiscitis tuberculosa o mal de Pott es la forma de afectación osteoarticular más frecuente $^{4}$. La infección comienza en el hueso subcondral y se extiende lentamente al espacio discal y a los cuerpos vertebrales adyacentes. Las vértebras torácicas inferiores y las lumbares superiores son las afectadas mayoritariamente, y también suele cursar con abscesos paravertebrales ${ }^{1,5}$. Los síntomas más frecuentes son dolor y rigidez de la columna que se exacerba con el movimiento y evoluciona de forma insidiosa durante meses. La 
resonancia magnética nuclear es muy sensible y útil para el diagnóstico presuntivo, pero se requiere la demostración de Mycobacterium tuberculosis, o de granulomas en muestras de biopsia o punciónaspiración con aguja fina (PAAF) procedentes de vértebras con lesiones compatibles, para confirmar la etiología ${ }^{1,4,5}$. La enfermedad avanzada puede dar lugar a deformidad y/o déficit neurológico ${ }^{7}$.

Con el fin de aportar nuestra experiencia para un mejor conocimiento de esta enfermedad, realizamos un estudio retrospectivo, clínico y microbiológico, de los casos de espondilodiscitis tuberculosa diagnosticados en el Área Sanitaria de Cádiz (España) durante un período de diez años.

\section{Material y Método}

Estudiamos seis pacientes con diagnóstico microbiológico de espondilodiscitis tuberculosa atendidos en el Hospital Universitario Puerta del Mar de Cádiz (España) durante los años 2000-2009. De estos pacientes se recogieron datos referentes a edad, sexo, prueba de Mantoux, localización de la enfermedad, síntomas de presentación, tiempo de evolución desde el inicio de los síntomas hasta el diagnóstico, presencia de abscesos paravertebrales, resultados de la tinción de Ziehl-Neelsen y del cultivo micobacteriano, sensibilidad de las cepas a los fármacos habituales, duración del tratamiento antibiótico y necesidad de cirugía.

El diagnóstico clínico se fundamentó en la presencia de raquialgia de ritmo inflamatorio, limitación de la movilidad y hallazgos radiológicos, de resonancia nuclear magnética (RNM) o ambos. El diagnóstico microbiológico requirió la toma de muestras mediante biopsia o PAAF guiada por TAC, que se procesaron el mismo día de su recepción. En todos los casos se realizó tinción de Ziehl-Neelsen y/o auramina, cultivo en medio sólido de Löwenstein-Jensen (Soria-Melguizo, España) y medio líquido de Middlebrook $7 \mathrm{H} 9$ procesado en el sistema MGIT 960 (Becton-Dickinson, Francia). La identificación de las cepas se efectuó por hibridación de ADN con sondas genéticas (Gen-Probe, Bio-Mèrieux, Francia) y el estudio de sensibilidad se llevó a cabo mediante el método de las proporciones de Canetti, Rist y Grosset, frente a isoniacida, estreptomicina, etambutol, rifampicina, etionamida y pirazinamida.

\section{Resultados}

Durante el período estudiado (2000-2009) se diagnosticaron 292 casos de infección tuberculosa comprobada por el Servicio de Microbiología de nuestro hospital; de ellos, 231 pacientes $(79,1 \%)$ desarrollaron tuberculosis pulmonar y 61 (20,9\%) afectación extrapulmonar de la enfermedad. En 8 de estos casos $(13,1 \%)$ hubo afectación ósea, de los que 6 correspondieron a espondilodiscitis. La espondilodiscitis tuberculosa supuso 15\% de todas las espondilodiscitis infecciosas diagnosticadas.

Las características de los pacientes con espondilodiscitis tuberculosa se resumen en la Tabla 1. El sexo femenino fue el más afectado (66,6\%), y la edad media fue de 54,3 años. Cinco pacientes fueron caucasianos y de nacionalidad española, y el sexto marroquí. Dos de los pacientes tenían antecedentes de infección por VIH y tres de tuberculosis pulmonar. La prueba de Mantoux fue positiva en tres pacientes. La radiografía simple de tórax sólo mostró hallazgos sugerentes de enfermedad pulmonar en dos de los casos. La localización fue lumbar en tres pacientes y dorsal en los restantes. La tinción de Ziehl-Neelsen fue positiva en tan sólo dos casos, mientras que el cultivo lo fue en todos.

El tiempo medio de evolución desde la presentación de los síntomas hasta el momento del diagnóstico fue de 7,3 meses (intervalo de 1 a 20 meses). El cuadro clínico se manifestó con dolor vertebral en todos los casos (irradiado a miembros inferiores en dos de ellos), fiebre en dos casos; tan sólo un paciente presentó síntomas neurológicos. Cuatro de los afectados mostraron abscesos de localización paravertebral; uno de ellos acompañado de absceso epidural y otro de absceso epidural y perivertebral. En 5 pacientes la velocidad de sedimentación globular fue elevada $(\geq 71 \mathrm{~mm}$ en la primera hora).

Todas las cepas fueron sensibles a los tuberculostáticos habituales. Los pacientes recibieron tratamiento específico de acuerdo con los resultados del antibiograma: 5 se trataron con isoniacida, rifampicina y pirazinamida, durante 12 meses, adicionando etambutol durante los dos primeros meses; el caso restante recibió rifampicina, isoniacida y etambutol. Tres de los pacientes requirieron tratamiento quirúrgico. La evolución fue favorable en 5 pacientes, sin presentar secuelas. 
Tabla 1. Características de 6 casos de espondilodiscitis tuberculosa en Cádiz (España), 2000-2009

\begin{tabular}{|c|c|c|c|c|c|c|c|c|c|c|c|}
\hline Caso & $\begin{array}{l}\text { Edad } \\
\text { /sexo }\end{array}$ & $\begin{array}{l}\text { Enferme- } \\
\text { dad de base }\end{array}$ & $\begin{array}{l}\text { Locali- } \\
\text { zación }\end{array}$ & PPD* & BAAR & $\begin{array}{l}\text { Cul- } \\
\text { tivo }\end{array}$ & AP & $\begin{array}{l}\text { Tiempo } \\
\text { diagnós- } \\
\text { tico }\end{array}$ & Cirugía & Tratamiento & Evolución \\
\hline 1 & $V / 43$ & VIH TBC & Dorsal & $P$ & $\mathrm{~N}$ & $P$ & $\mathrm{~N}$ & 6 meses & $\mathrm{Si}$ & $\begin{array}{l}\mathrm{INH}+\mathrm{RIF}+ \\
\mathrm{PZA}+\mathrm{ETB}\end{array}$ & Favorable \\
\hline 2 & $M / 42$ & - & Dorsal & $\mathrm{N}$ & $\mathrm{N}$ & $P$ & $P$ & 5 meses & No & $\begin{array}{l}\mathrm{INH}+\mathrm{RIF}+ \\
\mathrm{PZA}+\mathrm{ETB}\end{array}$ & Favorable \\
\hline 3 & $M / 56$ & - & Lumbar & $P$ & $P$ & $P$ & $P$ & 20 meses & $\mathrm{Si}$ & $\begin{array}{l}\mathrm{INH}+\mathrm{RIF}+ \\
\mathrm{PZA}+\mathrm{ETB}\end{array}$ & Favorable \\
\hline 4 & M/29 & $\begin{array}{c}\text { VIH VHC } \\
\text { TBC }\end{array}$ & Lumbar & $P$ & $P$ & $P$ & $P$ & 1 mes & $\mathrm{Si}$ & $\begin{array}{l}\mathrm{INH}+\mathrm{RIF}+ \\
\mathrm{PZA}+\mathrm{ETB}\end{array}$ & Favorable \\
\hline 5 & $\mathrm{M} / 80$ & TBC & Dorsal & $\mathrm{N}$ & $\mathrm{N}$ & $P$ & $P$ & 6 meses & No & $\begin{array}{l}\text { INH+RIF+ } \\
\text { ETB }\end{array}$ & Éxitus \\
\hline 6 & V/76 & - & Lumbar & $\mathrm{N}$ & $\mathrm{N}$ & $P$ & $\mathrm{~N}$ & 6 meses & No & $\begin{array}{l}\text { INH }+ \text { RIF+ } \\
\text { PZA +ETB }\end{array}$ & Favorable \\
\hline
\end{tabular}

${ }^{*}$ PPD $=$ Prueba de Mantoux; $\mathrm{P}=$ Positivo; $\mathrm{N}=$ Negativo; $\mathrm{AP}=$ Anatomía Patológica; INH = Isoniacida; RIF = Rifampicina; $\mathrm{PZA}=$ Pirazinamida; $\mathrm{ETB}=$ Etambutol.

\section{Discusión}

La incidencia de espondilodiscitis tuberculosa en el Área Sanitaria de Cádiz es menor que la publicada en otras regiones de España ${ }^{8,9}$. Sin embargo, si tenemos en cuenta la incidencia de tuberculosis respiratoria, superior en estas regiones en comparación con la nuestra ( 18 casos/100.000 habitantes en 2000 y 13 casos/ 100.000 habitantes en 2009), nuestra incidencia es similar. De todas formas, la incidencia de espondilodiscitis tuberculosa es subestimada en la mayoría de los estudios, debido probablemente al empleo de métodos epidemiológicos poco rigurosos aplicados a partir de series de casos de hospitales de referencia.

En las series recientes, las espondilodiscitis tuberculosas en inmigrantes suponen más de 50\% de los casos recogidos sin embargo, en nuestra serie sólo un paciente fue inmigrante $(17 \%)$. A diferencia de otros autores, encontramos una mayor frecuencia en mujeres, aunque la edad en los pacientes de nuestra serie es la habitual en países desarrollados ${ }^{2,8,10}$ Los datos clínicos sugieren en todos los estudios que la espondilodiscitis tuberculosa es un proceso crónico y lentamente progresivo, tal como comprobamos en nuestro estudio. Tan sólo dos pacientes de nuestra serie presentaron fiebre, ello junto a la pérdida de peso puede indicar habitualmente tuberculosis activa en otros órganos ${ }^{1}$, no obstante en nuestros pacientes no encontramos actividad en otra localización.

En la mayoría de los casos de espondilodiscitis tuberculosa sólo se afecta un segmento raquídeo, siendo las últimas vértebras dorsales y la columna lumbar las más involucradas²; ello coincide con nuestra casuística, donde en la mitad de los casos se vio afectada la columna dorsal y en la otra mitad, la región lumbar. La enfermedad intratorácica concurrente está presente en menos de 50\% de los casos ${ }^{9}$, lo cual concuerda con nuestros datos. A diferencia de otros estudios, que encuentran positividad de la prueba de Mantoux en casi la totalidad de los pacientes ${ }^{11,12}$, sólo la mitad de los nuestros reaccionaron a la tuberculina.

Las radiografías simples de la columna vertebral aportan escasa información en fases iniciales de la enfermedad, pero a partir de la octava semana comienza a objetivarse esclerosis reactiva con neoformación ósea. En la espondilodiscitis tuberculosa se producen lesiones osteolíticas y abscesos paravertebrales de mayor tamaño que en las no tuberculosas ${ }^{13}$.

El tratamiento de la espondilodiscitis tuberculosa es fundamentalmente médico y debe instaurarse lo más precozmente posible. Consiste en reposo, colocación de órtesis de descarga del 
segmento raquídeo afectado y la administración de los tuberculostáticos habituales para la infección tuberculosa pulmonar: isoniacida (300 mg/día), rifampicina $(600 \mathrm{mg} /$ día $)$, pirazinamida (1.500 $\mathrm{mg} /$ día) y/o etambutol (1.200 mg/día), si no se detecta resistencia o hipersensibilidad a alguno de estos fármacos ${ }^{8}$. En relación a la duración del tratamiento existen diferentes recomendaciones desde 6 a 12 meses, e incluso 15 meses $^{14}$. En nuestra serie la pauta empleada fue la habitual, durante 12 meses, más etambutol durante los dos primeros meses, obteniendo buena evolución clínica, sin complicaciones; el único paciente fallecido presentaba edad avanzada y enfermedades subyacentes que pudieron desencadenar el éxitus. La indicación quirúrgica se plantea cuando aparece deterioro neurológico agudo grave, inestabilidad o deformidad espinal, si no hay respuesta a quimioterapia o si el paciente incumple el tratamiento médico ${ }^{13}$.

\section{Referencias}

1. Fanlo P, Tiberio G. Tuberculosis extrapulmonar. An Sist Sanit Navar 2007; 30: 143-62.

2. García-Lechuz JM, Julve R, Alcalá L. Espondilodiscitis tuberculosa o enfermedad de Pott: experiencia en un hospital general. Enferm Infecc Microbiol Clin 2002; 20: 5-9.

3. Rubio B, Calvo B, García J, Ciria L, Navarro ML, Ramos Amador JT. Espondilodiscitis en la Comunidad de Madrid. An Pediatr (Bar) 2005; 62: 147-52.

4. Herrera A, Rodríguez J, Vicente J, Calvo A. Espondilodiscitis tuberculosa. Mal de Pott. Rev Ortop Traumatol 1998; 42: 231-42.
5. Fica A, Bozán F, Aristegui M, Bustos P. Espondilodiscitis. Análisis de una serie de 25 casos. Rev Med Chile 2003: $131 ; 473-82$.

6. Wirtz DC, Genios I, Wilderberg JE, Adam G, Zilkens KW, Niethard FU. Diagnostic and therapeutic management of lumbar and thoracic spondylodiscitis-an evaluation of 59 cases. Arch Orthop Trauma Surg 2000; 120: $245-51$

7. Alothman A, Memish ZA, Awada A, Al-Mahmood S, Al-Sadoon S, Rahman MM, et al. Tuberculous spondylitis: analysis of 69 cases from Saudi Arabia. Spine 2001; 26: 565-70.

8. Gómez N, Penelas-Cortés Y, Ibáñez J. Espondilodiscitis infecciosa en un área sanitaria gallega, 1983-2003. An Med Intern 2004; 11: 533-9.

9. Solís-García J, Vives M, Lizán M, Martínez E, Segura JC, Solera J. Incidencia de la espondilitis infecciosa en la provincia de Albatece. Enferm Infecc Microbiol Clin 2005; 23: 545-50.

10. Karadimas EJ, Bunger C, Lindblad BE, Hansen ES, Hoy $\mathrm{K}$, Helmig P, et al. Spondylodiscitis. A retrospective study of 163 patients. Acta Orthop 2008; 79: 650-9.

11. Perrone C, Saba J, Behloul Z. Pyogenic and tuberculous spondylodiskitis (vertebral osteomielitis) in 80 adults patients. Clin Infect Dis 1994; 19: 746-50.

12. Hayes AJ, Chocksey M, Barnes N, Sharrow O. Spinal tuberculosis in developed countries: difficulties in diagnosis. J R Coll Surg Edimb 1996; 41: 192-6.

13. Benavente AM, Monge MI, Acal P. Espondilodiscitis tuberculosa lumbar. Rehabilitación (Madr) 2004; 38 : 37-40.

14. Corti M, Villafañe MF, Yampolsky C, Ambroggi M, Palmieri O. Espondilodiscitis con absceso epidural espinal y del psoas por Mycobacterium tuberculosis. Rev Panam Infectol 2007; 9: 50-3. 


\section{Invaginación intestinal en adultos como manifestación de enfermedad celíaca. Caso clínico}

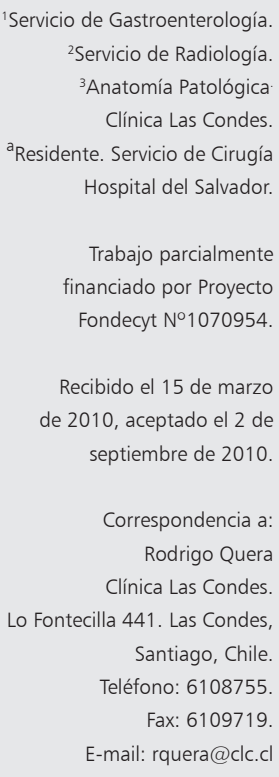

\author{
RODRIGO QUERA ${ }^{1}$, CLAUDIO HEINE T.a, \\ ANDRÉS O'BRIEN'², LUIS CONTRERAS ${ }^{3}$
}

\section{Celiac disease presenting as an intestinal intussusception. Report of one case}

\begin{abstract}
Acute abdominal pain caused by perforation, intestinal lymphoma or intussusception is an uncommon manifestation in adult celiac disease. We report a 49 yearold female with history of irritable bowel syndrome and osteoporosis consulting for acute abdominal pain and severe abdominal distention. Abdominal CT scan and magnetic resonance imaging showed a jejunal intussusception and other radiological alterations that suggested the possibility of celiac disease. Serological antibodies (endomysial and transglutaminase antibodies), endoscopy and the pathological study of duodenal biopsies confirmed the diagnosis. With a gluten free diet, the patient remains asymptomatic.
\end{abstract}

(Rev Med Chile 2010; 138: 1276-1280).

Key words: Celiac disease; Intussusception.
L a enfermedad celíaca (EC), una condición fuertemente asociada a los antígenos HLAtipo II DQ2 y DQ8 ${ }^{1}$, es definida como una enteropatía inmune provocada por una reacción al gluten ${ }^{2}$. Esta enfermedad de distribución global, puede ser diagnosticada a cualquier edad y afecta múltiples órganos y sistemas. Tiene una prevalencia mundial de alrededor de 1\%, sin embargo, se estima que ésta es aun mayor, ya que sólo 10\% de las personas afectadas son diagnosticadas ${ }^{3}$. En nuestro país no existen datos al respecto, pero es claro que su incidencia va en aumento, al igual que en el resto del mundo ${ }^{4}$.

La invaginación intestinal es un cuadro causado cuando un segmento del intestino (intususeptum) se introduce en el segmento distal adyacente del intestino (intususcipiens), siendo la causa más frecuente de obstrucción intestinal en niños $<5$ años. En estos casos, una lesión estructural del intestino es identificada solamente en el 5-15\% de los casos, y la mayoría de estos (> 80\%) se resuelve con un tratamiento conservador ${ }^{5}$. Por el contrario, en el adulto la invaginación intestinal es una patología infrecuente ya que representa sólo el 5\% de todos los casos de invaginación intestinal y $1-5 \%$ de las causas de obstrucción intestinal del adulto ${ }^{6}$. Estudios radiológicos recientes han demostrado que en pacientes adultos, sólo $30 \%$ de las invaginaciones intestinales presentan un punto de origen identificable y que sobre $50 \%$ de los pacientes sin este punto de origen identificable presentan una etiología idiopática ${ }^{7,8}$. Sin embargo, en su mayoría, estos casos no han sido seguidos de manera prolongada. Entre las posibles etiologías de las invaginaciones entero-entéricas sin un punto de origen destacan procesos inflamatorios (pancreatitis aguda, colecistitis, apendicitis), adherencias, enfermedad de Crohn, esclerodermia, fibrosis quística y EC 9 . Artículos, tanto en población pediátrica como adulta, han demostrado que la invaginación intestinal puede ser una forma de presentación de la $\mathrm{EC}^{10-14}$. Aunque ya existe 
Invaginación intestinal en enfermedad celíaca - R. Quera et al

un caso clínico a nivel nacional que muestra la invaginación intestinal como una manifestación de la $\mathrm{EC}^{13}$, creemos que nuestro caso confirma que esta etiología debe ser considerada en el diagnóstico diferencial de la invaginación intestinal sin un punto de origen, sobre todo si otros criterios radiológicos están presentes.

\section{Caso clínico}

Paciente de 49 años, sexo femenino, con antecedentes de síndrome intestino irritable tipo constipación desde la adolescencia, gastritis medicamentosa por uso de antiinflamatorios no esteroidales (AINE) el año 2000 (endoscopia digestiva alta duodeno normal), osteporosis el año 2004 y tiroiditis aguda el año 2005 en tratamiento con levotiroxina $75 \mathrm{mcg} / \mathrm{día}$. El año 2001 presentó cuadro de dolor abdominal en fosa iliaca derecha intenso, de inicio súbito, por lo que fue hospitalizada con diagnóstico de apendicitis aguda, la que se descartó por ecotomografía abdominal y una evolución clínica favorable. Dada la intensidad del cuadro clínico se realizó una colonoscopia que mostró la presencia de una compresión extrínseca a nivel cecal. Fue evaluada con TC de abdomen y pelvis donde se señaló que existía una masa en el anexo derecho que comprimía el ciego. Se decidió realizar una laparoscopia exploradora cuyo hallazgo fue la presencia de una distensión del colon derecho asociado a una profusa peristalsis del intestino delgado y del colon. Los ovarios eran normales y las trompas se apreciaban eritematosas y rígidas, por lo que se concluyó una salpingitis crónica bilateral, realizándose una salpingectomía bilateral. Las biopsias posteriores descartaron la presencia de cualquier proceso inflamatorio. $\mathrm{La}$ paciente se mantuvo en buenas condiciones generales presentando episodios aislados de malestar y distensión abdominal hasta agosto de 2009, fecha en que fue hospitalizada con diagnóstico probable de obstrucción intestinal por un nuevo cuadro de dolor abdominal agudo e intenso, asociado a distensión abdominal importante. Se realizó una TC de abdomen y pelvis donde destacaba la presencia de una lesión focal hepática sólida de un $\mathrm{cm}$ de diámetro en el segmento VI, no caracterizable en este estudio, además de múltiples áreas de invaginación en yeyuno, probablemente transitorias, no asociado a signos de obstrucción intestinal (Figura 1A -D). La paciente fue tratada en forma sintomática con buena respuesta y se dio de alta con indicación de estudio de resonancia magnética para evaluación de la lesión hepática. Este examen se realizó estando la paciente asintomática, describiéndose dos lesiones hepáticas de 3 y $10 \mathrm{~mm}$ en segmento IV y VI respectivamente, compatibles con hemangiomas, además de los siguientes ha-
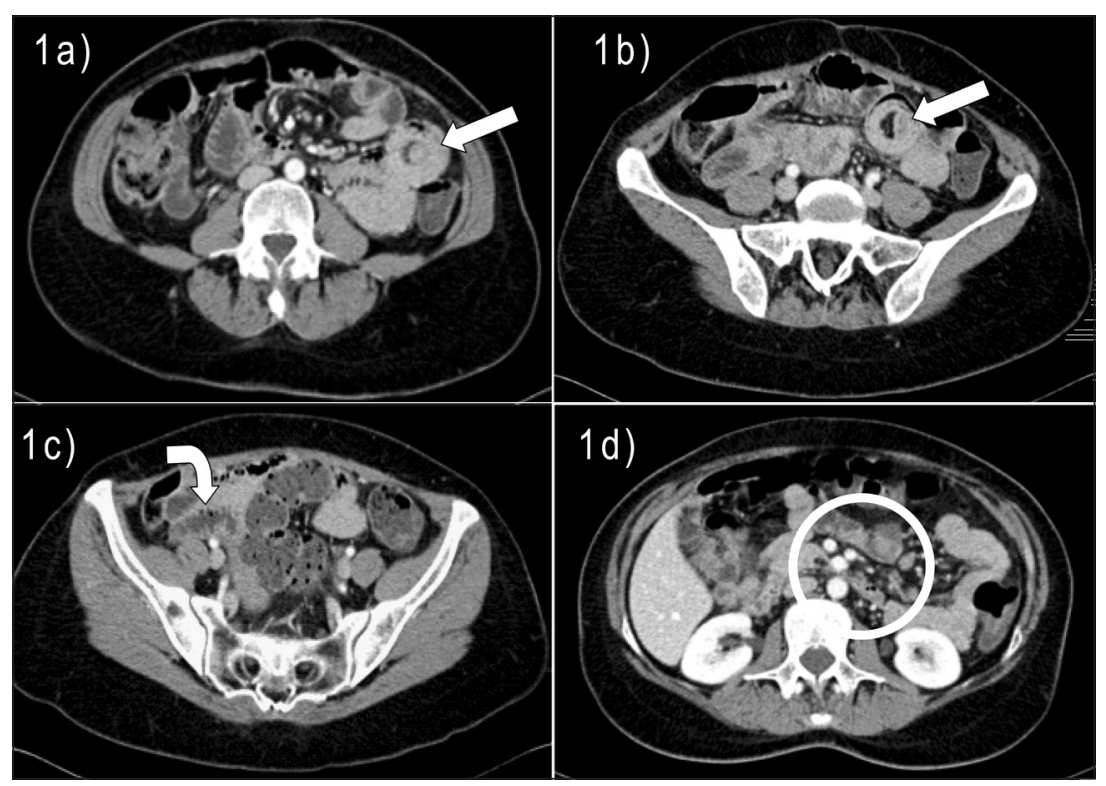

Figura 1. Alteraciones en TC de abdomen y pelvis (cortes axiales). 1a) y $1 \mathrm{~b}$ ): Signo de la crescente (grasa mesentérica invaginada) que confirma el diagnóstico de invaginación intestinal (flechas rectas). 1c): Aumento del número de pliegues a nivel de íleon (flecha curva). 1d): Pequeñas adenopatías mesentéricas (en círculo). 

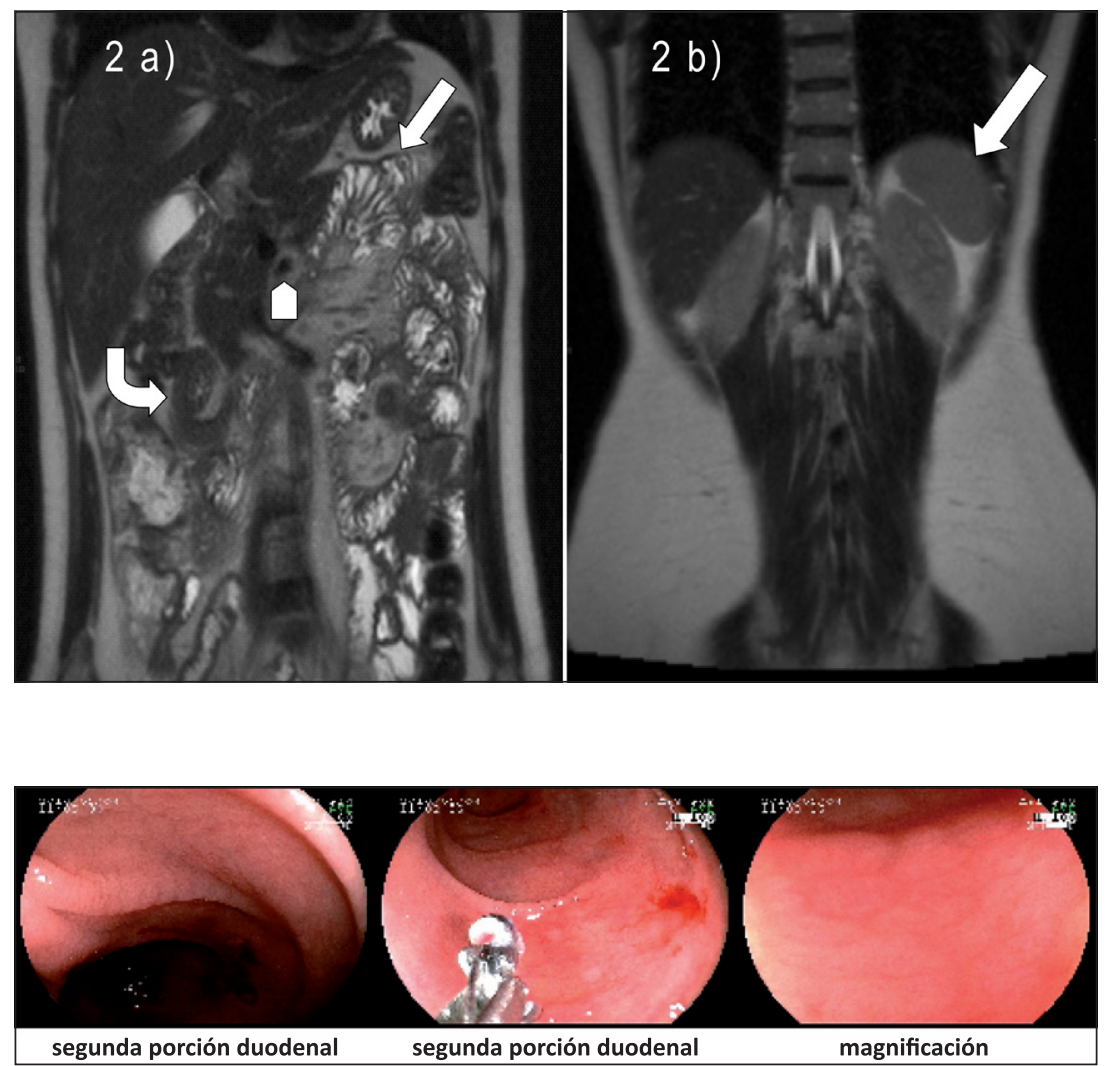

Figura 2. Alteraciones en la resonancia magnética de hígado (T2 HASTE coronal). 2a): Invaginación de asa de yeyuno en flanco derecho (flecha recta), asas de yeyuno distendidas en flanco izquierdo (flecha curva) y arteria mesentérica superior prominente (punta de flecha). 2b): Hipoesplenia (flecha recta).

Figura 3. Alteraciones a nivel de duodeno en la endoscopia digestiva alta. Pérdida difusa de las vellosidades intestinales con aspecto fenestrado y en ciertas áreas tipo empedrado a nivel de la segunda y tercera poción duodenal. llazgos: invaginación transitoria de un asa de yeyuno en flanco derecho, asas distendidas a nivel de yeyuno, aumento significativo en el número de pliegues a nivel de las asas de íleon, linfonodos mesentéricos prominentes, origen aberrante de la arteria mesentérica superior e hipoesplenia. Estos signos radiológicos plantearon la posibilidad de una EC (Figura 2A-C). Se solicitaron exámenes serológicos (anticuerpo antiendomisio positivo y anticuerpo antitransglutaminasa $67 \mathrm{U} / \mathrm{ml}$; valor positivo $>25 \mathrm{U} / \mathrm{ml}$ ). La endoscopia (Figura 3) e histología (Figura 4) confirmaron el diagnóstico de EC. La paciente inició tratamiento con restricción de gluten evolucionando hasta la fecha en buenas condiciones generales sin referir síntomas digestivos en la actualidad.

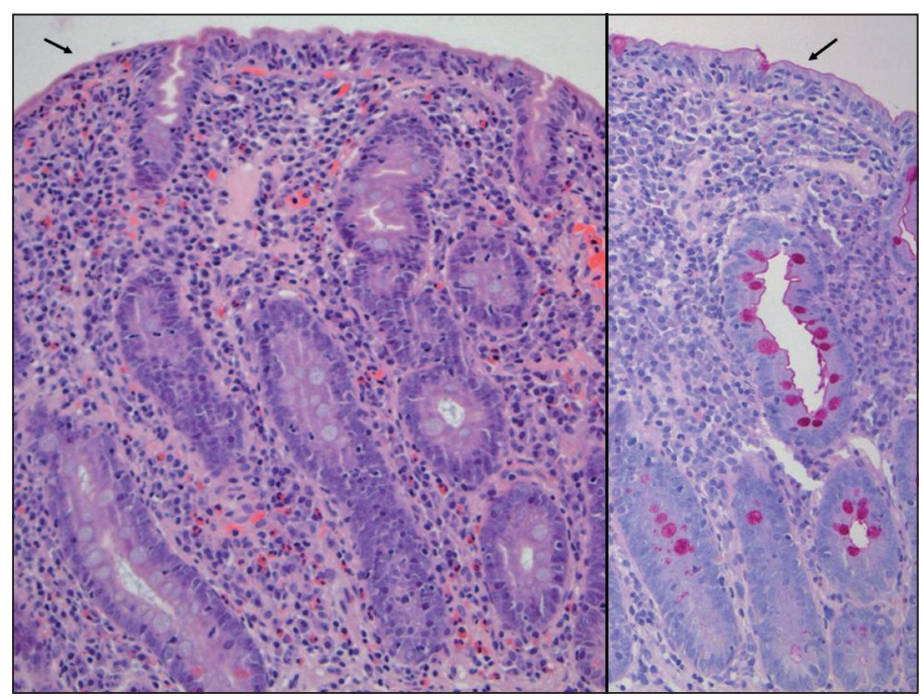

Figura 4. Alteraciones histológicas a nivel de duodeno. Marcada atrofia vellositaria con hipertrofia de las criptas e intensa linfocitosis intraepitelial (Marsh III-C). 


\section{Discusión}

La EC, una de las enfermedades gastrointestinales inmunes más frecuentes en Europa y Estados Unidos de Norteamérica, ha sido generalmente caracterizada como una enfermedad que suele presentarse en la infancia luego de la introducción del gluten. Sin embargo, estudios han demostrado que puede afectar a cualquier grupo etario y determinar una multiplicidad de síntomas clínicos ${ }^{3,15}$, situación que explica probablemente la demora en su diagnóstico. Las manifestaciones clínicas de la EC incluyen no sólo síntomas gastrointestinales inespecíficos como diarrea crónica, baja de peso o distensión abdominal, sino también síntomas secundarios a compromiso óseo (osteoporosis), dermatológico (alopecia, dermatitis herpetiforme), neurológico (ataxia, epilepsia) y reproductor (infertilidad) entre otros ${ }^{2,15}$.

La EC no diagnosticada puede ocasionalmente presentarse como un cuadro de urgencia ${ }^{16}$. Sanders y cols. mostraron que 3\% de los pacientes con dolor abdominal inespecífico presentaron el diagnóstico de EC. Sin embargo, este porcentaje aumentó a $10,5 \%$ al momento de considerar a aquellos pacientes con dolor abdominal no quirúrgico ${ }^{17}$. En pacientes con EC, el dolor abdominal puede ser causado por linfoma intestinal, adenocarcinoma intestinal, necrosis, perforación o invaginación intestinal. Distintas series y casos clínicos han demostrado la asociación entre EC e invaginación intestinal idiopática ${ }^{10-14}$. Recientemente, un estudio encontró que 11 de los 14 pacientes con EC e invaginación intestinal presentaron dolor abdominal al momento del diagnóstico ${ }^{14}$. Quizás el aporte más importante de nuestro artículo es confirmar que la invaginación intestinal puede ser una manifestación intestinal de la EC. Los mecanismos propuestos para el desarrollo de la invaginación intestinal en pacientes con EC, serían la presencia de inflamación a nivel de la mucosa asociado a la disfunción reversible del sistema nervioso extrínseco, lo que produciría una disminución de la motilidad a nivel del intestino delgado ${ }^{18,19}$. Estudios han sugerido que la presencia de invaginación intestinal se asocia a una mayor severidad de la atrofia de las vellosidades, pacientes con EC refractaria tipo II y linfoma células T asociado a enteropatía ${ }^{14,20}$. Tanto en el caso clínico publicado por Dodds y cols, como el nuestro, el paciente presentó un diagnóstico de EC Marsh
III-C ${ }^{13}$. Por otra parte, aunque la mayoría de los casos de invaginación intestinal en pacientes con EC no presentan un punto de origen, el adenocarcinoma y linfoma intestinal son complicaciones que pueden ocurrir en estos pacientes y deben ser consideradas al momento de determinar las posibles causas de la invaginación ${ }^{15,21}$.

La observación que la invaginación intestinal puede estar presente en pacientes con EC, evaluados por ecotomografía abdominal, TC de abdomen y pelvis o resonancia ${ }^{22-23}$, sugiere que estos pacientes pueden sufrir invaginaciones intestinales de manera frecuente y transitoria. Tomei y cols mostraron que en un grupo de 28 pacientes con EC, las alteraciones radiológicas más frecuentes en el TC de abdomen y pelvis fueron: alteración en el patrón de los pliegues intestinales $82 \%$, dilatación intestinal $75 \%$, aumento del líquido intestinal $64 \%$, linfoadenopatías $43 \%$, prominencia de los vasos mesentéricos $25 \%$, engrosamiento de la pared intestinal $21 \%$, invaginación intestinal $21 \%$, aumento del contenido intestinal de aire $14 \%$ y ascitis $7 \%{ }^{24}$. Estas mismas alteraciones han sido descritas utilizando la resonancia magnética como método de evaluación radiológica ${ }^{24}$. En nuestro paciente se encontraron seis de estos criterios.

Finalmente, dos puntos nos parecen importantes. Primero, que la EC debe ser considerada al momento de evaluar a pacientes con osteoporosis $^{25}$, sobre todo si esta se presenta a una edad temprana, como es el caso de nuestra paciente. Segundo, que la EC debe ser considerada en el diagnóstico diferencial del síndrome de intestino irritable ${ }^{26}$. Ambas situaciones estaban presentes en nuestro paciente antes que presentara el cuadro de invaginación intestinal y se diagnosticara la EC.

En conclusión, nosotros confirmamos que el dolor abdominal agudo puede ser una manifestación clínica en pacientes con EC. La invaginación idiopática, sobre todo si está asociada a otras alteraciones radiológicas, debe plantear la posibilidad de una EC. Teniendo esta manifestación clínica en mente será posible disminuir el número de paciente con EC no diagnosticada.

\section{Referencias}

1. Green PH, Cellier C. Celiac disease. N Engl J Med 2007; 357: 1731-43.

2. Herrera MJ, Hermosos M, Quera R. Enfermedad celíaca 
y su patogenía. Rev Med Chile 2009; 137: 1617-26.

3. Rewers M. Epidemiology of celiac disease: what are the prevalence, incidence, and progression of celiac disease? Gastroenterology 2005; 128: 547-51.

4. Lohi S, Mustalahti K, Kaukinen K, Laurila K, Collin $\mathrm{P}$, Rissanen $\mathrm{H}$, et al. Increasing prevalence of celiac disease over time. Aliment Pharmacol Ther 2007; 26: 1217-25.

5. Huppertz HI, Soriano-Gabarro M, Grimprel E, Franco E, Mezner Z, Desselberger U, et al. Intussusception among Young in Europe. Pediatr Infect J 2006; 25: S22-9.

6. Marinis A, Yiallourou A, Samanides L, Dafnios N, Anastasopoulos G, Vassiliou I, et al. Intussusception of the bowel in adults. A review. World J Gastroenterol 2009; 15: 407-11.

7. Lvoff N, Breiman RS, Coakley FV, Lu Y, Warren RS. Distinguishing features of self-limiting adult small-bowel intussusceptions indentified at CT. Radiology 2003; 227: 68-72.

8. Kim YH, Blake MA, Harisinghani MG, Archer-Arroyo K, Hahn PF, Pitman MB, et al. Adult intestinal intussusceptions: CT appearances and identification of a causative lead point. Radiographics 2006; 26: 733-4.

9. Agha FP. Intussusception in adults. AJR Am J Roentgenol 1986; 146: 527-31.

10. Martínez G, Israel NR, White JJ. Celiac disease presenting as entero-enteral intussusception. Pediatric Surg Int 2001; 17: 68-70.

11. Fishman DS, Chumpitazi BP, Ngo PD, Kim Hb, Lightdale JR. Small bowel intussusception in celiac disease: Revisiting a classic association. J Pediatr Gastroenterol Nutr 2010; 50: 237.

12. Makay O, Kazimi M, Doğanavşargil B, Osmanoğlu N, Yilmaz M. Acute abdomen in adult celiac disease: As intestinal intussusception case. Turk J Gastroenterol 2007; 18: 103-6.

13. Dodds BF, Aguancha SI, Santamarina RM, Vega SJ. Obstrucción intestinal por intususcepción como primera manifestación de enfermedad celíaca en adulto. Rev Med Chile 2008; 136: 1179-82.

14. Gonda TA, Khan SU, Cheng J, Lewis SK, Rubin M, Green $\mathrm{PH}$. Association of intussusception and celiac disease in adults. Dig Dis Sci 2009 Diciembre 24. Epub ahead of print.
15. Tack GI, Verbbek WH, Schreurs MW, Mulder CJ. The spectrum of celiac disease: epidemiology, clinical aspects and treatment. Nat Rev Gastroenterol Hepatol 2010; 7: 204-13.

16. Sanders DS, Hurlstone DP. Do patients with unrecognized coeliac disease presents as an emergency?. Eur J Emerg Med 2005; 12: 303-5.

17. Sanders DS, Hopper AD, Amzy IA, Rahman N, Hurlstone DP, Leeds JS, et al. Association of adult celiac disease with surgical abdominal pain: a case-control study in patients referred to secondary care. Ann Surg 2005; 242: 201-7.

18. Bassotti G, Villanacci V, Mazzocchi A, Mariano M, Incardona P, Clerici C, et al. Antroduodenojejunal motor activity in untreated and treated celiac disease patients. J Gastroenterol Hepatol 2008; 23: e23-8.

19. Bassotti G, Castellucci G, Betti C, Fusaro C, Cavalletti ML, Bertotto A, et al. Abnormal gastrointestinal motility in patients with celiac sprue. Dig Dis Sci 1994; 39: 1497 54.

20. Garrido A, Luque A, Vázquez A, Hernández JM, Alcántara F, Márquez JL. Primary small bowel neoplasms as a complication of celiac disease. Gastroenterol Hepatol 2009; 32: 618-21.

21. Mallant M, Hadithi M, Al-Toma AB, Kater M, Jacobs M, Manoliu R, et al. Abdominal computed tomography in refractory coeliac disease and enteropathy associated T-cell lymphoma. World J Gastroenterol 2007: 13; 1696700.

22. Bartusek D, Valek V, Husty J, Uteseny J. Small bowel ultrasound in patients with celiac disease. Retrospective study. Eur J Rad 2007; 63: 302-6.

23. Tomei E, Diacinti D, Marini M, Mastropasqua M, Di Tola M, Sabbatella L, et al. Abdominal CT findings may suggest coeliac disease. Dig Liver Dis 2005; 37: 402-6.

24. Tomei E, Semelka R, Braga L, Laghi A, Paolantonino P, Marini M, et al. Adult celiac disease: what is the role of MRI?. J Magn Reson Imaging 2006; 24: 625-9.

25. Duerksen DR, Leslie WD. Positive celiac disease serology and reduced bone mineral density in adult women. Can J Gastroenterol 2010; 24: 103-7.

26. Jadallah KA, Khader YS. Celiac disease in patients with presumed irritable bowel syndrome: a case-finding study. World J Gastroenterol 2009;15: 5321-5. 